\title{
TYPIFICATION AND CORRECTION OF SYNTAXA FROM THE CLASS MOLINIO-ARRHENATHERETEA TX. 1937 IN SERBIA
}

\author{
Svetlana AĆIĆ́ ${ }^{1}$, Urban ŠILC, ${ }^{2,4}$, Dmitar LAKUŠIĆ ${ }^{3}$, Snežana VUKOJIČIĆc ${ }^{3}$ \& \\ Zora DAJIĆ STEVANOVIĆ ${ }^{1}$
}

\begin{abstract}
A list of corrected and typified grassland communities of the class Molinio-Arrhenatheretea Tx. 1937 occurring in Serbia was provided. The nomenclature rules of the International Code of Phytosociological Nomenclature were strictly followed. Syntaxonomic affiliation of communities to higher syntaxa was assessed according to the existing syntaxonomical schemes for Serbia, i.e. according to the position determined by the original source. Higher syntaxa followed the synsystem of the so-called "EuroVegChecklist". Since syntaxonomic disagreements concerning a certain plant association's position within the whole classification system have not been discussed, such a list should serve as the starting point for a further revision of the status of grassland communities in Serbia. So far, a total of 87 plant communities of the class Molinio-Arrhenatheretea were described for Serbia.

The list provided here will enable more precise and more accurate mapping of vegetation in Serbia, as well as classification of these communities into the Habitat Directive which will enable the establishment of the Red list of Habitats for Serbia.

Key words: phytosociology, syntaxonomy, International Code of Phytosociological Nomenclature.

\section{Izvleček}

V članku so predstavljene nomenklaturno popravljene in tipifizirane traviščne združbe razreda Molinio-Arrhenatheretea Tx. 1937, ki se pojavljajo v Srbiji. Dosledno smo upoštevali pravila Mednarodnega kodeksa fitocenološke nomenklature. Sintaksonomsko pripadnost združb višjim sintaksonom smo ohranili glede na obstoječe sintaksonomske sheme v Srbiji oziroma glede na uvrstitev v originalnem viru. Višji sintaksoni sledijo sintaksonomiji po "EuroVegChecklist". Glede na to, da nismo obravnavali uvrstitve posameznih rastlinskih združb v sintaksonomski sistem, bo ta seznam omogočil sintaksonomsko revizijo traviščnih združb Srbije. Do sedaj je bilo na ozemlju Srbije opisanih 87 rastlinskih združb razreda Molinio-Arrhenatheretea. Seznam bo omogočil tudi bolj natančno kartiranje vegetacije Srbije in povezavo teh združb s Habitatno direktivo in izdelavo Rdečega seznama habitatnih tipov Srbije.
\end{abstract}

Ključne besede: fitosociologija, sintaksonomija, Mednarodni kodeks fitocenološke nomenklature.

\section{INTRODUCTION}

Phytocoenological studies of vegetation of Serbia encompassing floristic, ecological and other characteristics of plant communities started more than 70 years ago (Sarić 1984). Situated in the Balkan Peninsula, Serbia is characterized by diverse climatic, edaphic, geomorphological and phytogeographical conditions causing, along with long-lasting human impact, the occurrence of complex meadow and pasture vegetation.

The first vegetation studies in Serbia were conducted by Josif Pančić in 1846 and were continued with research of Balkans vegetation by $\mathrm{Lu}-$ jo Adamović from 1909. Researchers from Serbia used the methodology of the French-Swiss phy-

\footnotetext{
${ }^{1}$ University of Belgrade, Faculty of Agriculture, Nemanjina 6, Belgrade-Zemun, Serbia; acic@agrif.bg.ac.rs

${ }^{2}$ Institute of Biology ZRC SAZU, Novi Trg 2, Ljubljana, Slovenia

${ }^{3}$ University of Belgrade, Faculty of Biology, Institute of Botany and Botanical Garden, Takovska 43, Belgrade, Serbia

${ }^{4}$ BC Naklo, Strahinj 99, Naklo, Slovenia
} 
tocoenological school of Braun-Blanquet (1928). Igor Rudsky was the first scientist from Serbia using Braun-Blanquet's method in vegetation studies that started already before the Second World War (Rudskij 1934, Rudski 1938, Dinić et al. 2010).

The most significant progress in vegetation research was made in the period after the Second World War until the early 1990's when field studies stagnated due to the political and economic crisis and transition. Previous intensive phytosociological investigations led to the description and definition of a large number of plant communities of different vegetation classes, as well as to the elaboration of a syntaxonomical system for the vegetation of Serbia.

The syntaxonomy and diversity of the vegetation of the territory of the western Balkans were first considered by the Vegetation map of Yugoslavia (Jovanović et al. 1986) and Prodromus phytocoenosum Jugoslaviae (Zupančič et al. 1986), Prodromus of vegetation of Serbia excluding territories of provinces (Jovanović \& Jovanović 1976), Prodromus of vegetation of Kosovo (Ranđelović \& Rexhepi 1980) and Prodromus of vegetation of Vojvodina (Parabućski et al. 1986). Unfortunately, these reviews are not sufficiently detailed and precise and do not encompass the entire herbaceous vegetation, including particular meadows and pastures. The next, much more comprehensive portrait of Serbian vegetation was the "Syntaxonomical Review of Vegetation of Serbia" (Kojić et al. 1998) in which grassland communities were presented in more detail. Nevertheless, this review failed to follow the recommendations of the Code of Phytosociological Nomenclature (Barkman et al. 1976, 1986), omitting standardization and nomenclatural correction of plant associations. Grassland vegetation of Serbia is presented in the monograph "Meadows and pastures of Serbia" (Kojić et al. 2004), which established the presence of 273 associations from 46 vegetation alliances and 10 classes. The characteristics of 10 broadly understood grassland associations was described in detail.

Very important are the results obtained from the Project "Harmonization of National Habitats Classification Nomenclature with European Community Standards" (Lakušić et al. 2005, Lakušić \& Sabovljević 2005). However, more attention was paid to habitats than to the nomenclature of communities.

Serbian botanists have more or less compiled the rules of the Code of Phytosociological Nomenclature (Barkmann et al. 1976, 1986), but there had been no previous practice of attaching the nomenclature type to the description of a new community. All communities described since 1979 are therefore invalid. So far, there have been no attempts in Serbia aimed at the correction and standardization of community denomination according to the International Code of Phytosociological Nomenclature (Weber et al. 2000; hereinafter: ICPN); hence, the present work could be considered as the first report in this area.

Our main goal was to check the validity and legitimacy of the existing nomenclature according to the ICPN and correct and typify the names of grassland communities belonging to the class Molinio-Arrhenatheretea in Serbia. The next step will be associated with appropriate positioning of these community types in supraregional classification schemes.

\section{METHODS}

The vegetation database of grassland vegetation of Serbia (Aćić et al. 2012) was the starting point for creating a list of grassland communities belonging to the class Molinio-Arrhenatheretea in Serbia. An extensive survey of relevant literature sources allowed us to check the validity and legitimacy of the nomenclature of grassland associations belonging to this class. The nomenclature rules of the ICPN were strictly followed. Affiliation of plant communities to their alliances and higher syntaxa followed the original source and Mucina et al. (in review), respectively.

The nomenclature of plant taxa follows the Flora Europaea (Flora Europaea Database), except for the moss species Brachythecium rivulare where the nomenclature follows Corley et al. (1981). Syntaxa were nominated according to their orthographically correct form according to ICPN Art. 41. The original name is provided in brackets with an indication of the relevant ICPN Article (indicated as Art.) according to which they are invalid or illegitimate.

All synonyms older than the respective accepted name include a reference to the article or paragraph of the Code according to which the name has to be rejected as ineffective, invalid or illegitimate.

Initials of the first author's names are added whenever necessary to avoid a homonymy. The 
dates of effective publication of syntaxa were deduced from the dates in the journals in which they were validly published, regardless of eventual different indications reported by the authors in the papers. Doctoral or degree theses, which are generally not available to libraries accessible to botanists, were not considered as effective publications [Art. 1]. In that case, we published a relevé as the holotype and a short description of the habitat of the association.

For the determination of correct names for syntaxa not yet typified lectotypes or neotypes were defined.

There was no intention to resolve association names which are frequently used in Serbian syntaxonomic overviews originating from other regions.

The accepted names of associations and alliances that contained only the genus name(s) in the original diagnosis were supplemented with species epithets in accordance with Recommendation 10c of the Code. In some cases, for practical reasons the modified form of names subject to approval by the Nomenclature Commission of the International Association for Vegetation Science was used. The form used is considered as a proposal for name modification. This concerns nomina inversa. According to Article 42 of the Code, nomina inversa are the names of syntaxa in which, as compared with the original diagnosis, the order of the names of taxa was changed so that the dominant taxon or the taxon of the higher layer is in the second place.

According to Article 43 of the Code, the name of a syntaxon must be corrected due to taxonomic errors when the name of an aggregate species is replaced by the name of a narrowly defined species.

A 'phantom name' is a name that has not been used by the person(s) named in the author citation in the stated source, either literally or in a form which is homonymous according to the regulations of ICPN. The 'cited' source may even be non-existent. Mucina (1993) introduced the striking term 'phantom name' for such cases. They are attributions by later authors and do not have any nomenclatural significance.

Despite our comprehensive overview of the relevant phytosociological literature, it is still possible that another, older and valid name will be appointed as relevant for some syntaxa in future phytosociological literature, and this name will have to be accepted as the correct name.
Abbreviations: Art. = article of ICPN; nom. corr. = nomen correctum; nom . ined. $=$ nomen ineditum; nom. invers. propos. $=$ nomen inversum propositum; nom. mut. prop. $=$ nomen mutatum proposi tum; nom. nud. = nomen nudum; ass. nov. = associatio nova; nom. inval. $=$ nomen invalidum; Tab. $=$ phytocoenological table in publication; rel. = relevé.

\section{RESULTS AND DISSCUSION}

The list of corrected and typified grassland communities of the class Molinio-Arrhenatheretea occurring in Serbia:

\section{Molinio-Arrhenatheretea Tx. 1937}

\subsection{Molinietalia Koch 1926}

\subsubsection{Molinion caeruleae Koch 1926}

1. Caltho palustris-Alopecuretum pratensis $\mathrm{Bu}-$ torac et Hulo ex Aćić et al. ass. nov. hoc loco (Orig. Caltho laetae-Alopecuretum pratensis $\mathrm{Bu}-$ torac et Hulo 1993 nom. inval.) [Art. 5]

Typus: Butorac \& Hulo 1993, Tab. 2, rel. 2 holotypus hoc loco

\section{Carici gracilis-Poetum palustris Ilijanić 1968}

Note: This association was described for Serbia in Čapaković (1979).

\section{Scirpo holoschoeni-Salicetum rosmarinifoliae} Stjepanović-Veseličić 1953 nomen mutatum propositum

(Orig. Salicetum rosmarinifoliae StjepanovićVeseličić 1953, Asoc. Salix rosmarinifolia-Holoschoenus vulgaris Stjepanović-Veseličić 1953) [Art. 41b, 45]

Typus: Stjepanović-Veseličić 1953, Tab. 6, rel. 3 - lectotypus hoc loco

4. Lathyro pannonici-Molinietum caeruleae Tatić et al. ex Aćić et al. ass. nov. hoc loco (Orig. Lathyreto-Molinietum coeruleae Tatić et al. 1988 nom. inval.) [Art. 5]

Typus: Tatić et al. 1988, Table on page 33, rel. 5 - holotypus hoc loco

Note: There is some confusion as to the year of printing, because of the indication "1977 (1988)", which means that the year of printing was 1988. 


\section{Molinietum caeruleae W. Koch 1926}

Note: This community was described for Serbia in Stjepanović-Veseličić (1953), Kojić \& Ivanović (1953), Cincović \& Kojić (1956), Tatić (1969), Parabućski \& Butorac (1988) and Butorac \& Hulo (1993).

6. Molinio caeruleae-Deschampsietum cespitosae Pavlović 1951

(Orig. Molinieto-Deschampsietum Pavlović 1951) [Art. 41b]

Typus: Pavlović 1951, Tab. 11, rel. 2 - lectotypus hoc loco

\subsubsection{Calthion palustris Tx. 1937}

7. Brachythecio rivularis-Menthetum longifoliae V. Ranđelović et Zlatković ex Aćić et al. ass. nov. hoc loco

(Orig. Brachythecio-Menthetum longifoliae V. Ranđelović 2001 nom. inval.) [Art. 5]

Typus: Ranđelović \& Zlatković 2010, Tab. 22, rel. 5 - holotypus hoc loco

Note: The association was described in the doctoral thesis of V. Ranđelović (2002) and subsequently printed in the monograph (Ranđelović \& Zlatković 2010), but without an indication of the nomenclatural type.

8. Scirpetum sylvatici Ralski 1931

(syn. Polygono-Scirpetum sylvaticae Schwickerath 1944, Scirpetum sylvaticae Knapp 1946, EquisetoScirpetum silvatici prov. R. Jovanović 1969, Equiseto palustris-Scirpetum sylvatici Šegulja 1974)

Note: The association Equiseto palustris-Scirpetum sylvatici was described for Serbia in Ranđelović \& Zlatković (1994), and PolygonoScirpetum sylvaticae in Petković (1983) and Ranđelović \& Zlatković (1994).

9. Equisetetum palustris Danon et Blaženčić 1965

(Orig. Equisetetum palustrae Danon et Blaženčić 1965) [Art. 41a]

Typus: Danon \& Blaženčić 1965, Tab. 2, rel. 1 - lectotypus hoc loco

10. Cirsio palustris-Eriophoretum latifolii B. Jovanović 1969 nomen ineditum
(Orig. Cirsio palustre-Eriophoretum latifolii B. Jovanović 1969 [Art. 1])

Note: This association has never been published, but the data was taken from the reports for the mapping project of vegetation of Serbia (Jovanović B. et al. 1969). The name has not been published in a printed publication and is therefore rendered ineffective (nomen ineditum).

11. Equiseto palustris-Eriophoretum latifolii Petković ex Aćić et al. ass. nov. hoc loco

(Orig. Equiseto-Eriophoretum latifoli prov. Petković 1983 nom. inval.) [Art. 3b, 5]

Typus: Petković 1983, Tab. 4, rel.1 - holotypus hoc loco

\section{Selaginello selaginoidis-Eriophoretum latifolii} Petković et al. ex Aćić et al. ass. nov. hoc loco (Orig. Selaginello-Eriophoretum latifoli Petković et al. 1996 nom. inval.) [Art. 5]

Typus: Petković et al. 1996, Tab. 1, rel. 1 - holotypus hoc loco

\section{Sparganio polyedrae-Eriophoretum latifolii} Jovanović-Dunjić 1979 nomen invalidum (Orig. Sparganio-Eriophoretum latifolii R. Jovanović 1976 nom. inval.) [Art. 2b]

Note: The name of this syntaxon was not validly published because only a synoptic table was provided (Jovanović-Dunjić 1979). No single relevé is available as the nomenclatural type. Although the author states the year 1976, the year of printing was 1979.

\subsubsection{Deschampsion cespitosae Horvatić 1930}

\section{Agrostio stoloniferae-funcetum effusi Cincović} 1959

(Orig. Agrostideto-funcetum effusi Cincović 1959) [Art. 41b]

Typus: Cincović 1959, Tab. 1, rel. 10- lectotypus hoc loco

\section{Caricetum tricostatae-vulpinae Horvatić 1930}

Note: This association was described for Serbia in Babić (1955), Cincović (1956) and Danon (1960). 
16. Funco articulati-Deschampsietum cespitosae Petković ex Aćić et al. ass. nov. hoc loco (Orig. Funco-Deschampsietum caespitosae prov. Petković 1983 nom. inval.) [Art. 3b, 5] Typus: Petković 1983, Tab. 7, rel. 3 - holotypus hoc loco

\section{Deschampsietum cespitosae Horvatić 1930}

Note: This association was described for Serbia in Babić (1955), Gajić (1986), Purger (1993) and Ranđelović (2002).

18. Festucetum pratensis Gajić 1989 nomen nudum (Orig. Festucetum pratensis Gajić 1989 nomen nudum) [Art. 2]

Note: The name of the syntaxon is not validly published as there was no published phytocoenological table or relevé (Gajić 1989).

19. Rhinantho borbasii-Festucetum pratensis $\mathrm{Ga}$ jić ex Aćić et al. ass. nov. hoc loco

(Orig. Rhinantho-Festucetum pratensis Gajić 1986 nom. inval.) [Art. 5]

Typus: Gajić 1986, Tab. 5, rel. 1 - holotypus hoc loco

\section{Trifolio hybridi-Agrostietum stoloniferae Veljo-} vić 1967

(Orig. Trifolio-Agrostidetum Veljović 1967) [Art. 41b]

Typus: Veljović 1967a, Tab. 4, rel. 10 - lectotypus hoc loco

\subsection{Arrhenatheretalia elatioris Tx. 1931}

\subsubsection{Arrhenatherion elatioris Luquet 1926}

\section{Rumici acetosellae-Agrostietum caninae Cin-} cović 1959

(Orig. Rumiceto-Agrostidetum caninae Cincović 1959) [Art. 41b]

Typus: Cincović 1959, Tab. 4, rel. 8 - lectotypus hoc loco

22.Cynosuro cristati-Agrostietum stoloniferae Danon et Blaženčić in Mišić et al. ex Aćić et al. ass. nov. hoc loco

(Orig. Cynosuro-Agrostidetum albae prov. Danon et Blaženčić 1978 nom. inval.) [Art. 3b]
Typus: Mišić et al. 1978, Tab. 44, rel. 6 - holotypus hoc loco

23. Cynosuro-Agrostietum vulgaris A. Marković 1986 Phantom name

Note: Unknown reference used in Kojić et al. (2004).

\section{Festuco-Agrostietum capillaris Horvat 1962}

Note: This association was found in Serbia by Mišić et al. (1978), Petković (1985), JovanovićDunjić R. \& Jovanović S. (1991).

\section{Salvio verticillati-Agrostietum capillaris Gajić} ex Aćić et al. ass. nov. hoc loco

(Orig. Agrostidetum montanum Gajić 1961 nom. inval.) [Art. 34a]

Typus: Gajić 1961, Tab. 26, rel. 13 - holotypus hoc loco

26. Rhinantho alectorolophi-Agrostietum capillaris Danon et Radmić 1962 nomen mutatum propositum

(Orig. Alectorolopho-Agrostidetum vulgare Danon et Radmić 1962) [Art. 41b, 45]

Typus: Danon \& Radmić, 1962, Tab.3, rel. 2 lectotypus hoc loco

Note: Affiliation of associations to alliances followed the original source. Further research of the syntaxonomical position of associations dominated by Agrostis capillaris is required because these communities were classified into different classes (Molinio-Arrhenatheretea, Festuco-Brometea and Calluno-Ulicetea) by different Serbian authors (Aćić et al. 2012).

27. Ononido spinosiformis-Alopecuretum pratensis Butorac ex Aćić et al. ass. nov. hoc loco (Orig. Ononido-Alopecuretum pratensis Butorac 1992 nom. inval.) [Art. 5]

Typus: Butorac 1992, Tab. 7, rel. 2 - holotypus hoc loco

28. Ononido arvensis-Arrhenatheretum elatioris (Tomažič \& Horvatić 1941) Ilijanić \& Šegulja 1983

Note: This association was described for Serbia in Stojanović (1986). 
29. Pastinaco-Arrhenatheretum elatioris Passarge 1964

(syn. Arrhenatheretum medioeuropaeum Scherr. 1925 (non Br.-Bl. 1915) H-ić 1941)

Note: This association was described for Serbia in Slavnić (1948), Parabućski et al. (1982), Stojanović S. (1983), Jovanović R. (1983, 1986), Vučković R. (1985) and Vučković M. (1991).

\section{Ranunculo bulbosi-Arrhenatheretum elatioris} Ellmauer in Ellmauer \& Mucina 1993

Note: This association was first mentioned for Serbia by Aćić et al. (2013) and the association described as Arrhenatheretum elatioris Tüxen 1937 by Gajić et al. (1992) corresponds to this association.

While the description of the communities dominated by Arrhenatherum elatius for the province of Vojvodina used the name $A r$ rhenatheretum medioeuropaeum, the name $A r$ rhenatheretum elatioris was used for central Serbia. This wide and complex association (Arrhenatheretum elatioris Tüxen 1937) was reclassified into three ecologically narrower syntaxa by Ellmauer \& Mucina (1993). Thus, the former nomination of Arrhenatheretum medioeuropaeum corresponds to PastinacoArrhenatheretum elatioris, while in the central Serbia there are generally drier variants of the community characterized by high presence of species of the class Festuco-Brometea, corresponding to the ass. Ranunculo bulbosiArrhenatheretum elatioris.

31. Salvio nemorosae-Arrhenatheretum elatioris Parabućski ex Aćić et al. ass. nov. hoc loco (Orig. Salvio-Arrhenatheretum elatioris Parabućski 1990 nom. inval.) [Art. 5]

Typus: Parabućski 1990, Tab. 1, rel. 6 - holotypus hoc loco

32. Trifolio arvense-Brometum racemosi Danon et Blaženčić 1965

(Orig. Trifolieto-Brometum racemosi Danon et Blaženčić 1965) [Art. 41a]

Typus: Danon \& Blaženčić 1965, Tab. 2, rel. 2 - lectotypus hoc loco

33. Elymo repentis-Cynodontetum dactyloni Matović ex Aćić et al. ass. nov. hoc loco
(Orig. Agropyro-Cynodenetum dactyloni Matović 1986 nom. inval.) [Art. 5]

Typus: Matović 1986, Tab. 16, rel. 2 - holotypus hoc loco

34.Diantho deltoidis-Festucetum nigrescentis Pavlović 1951 corr. Aćić et al. hoc loco nomen inversum propositum

(Orig. As. Festuca fallax-Dianthetum deltoides Pavlović 1951) [Art. 41b, 42, 43]

Typus: Pavlović 1951, Tab. 9, rel. 4 - lectotypus hoc loco

Note: When a name of an association is formed from names of two taxa of which one is dominant then the name of that taxon appears on the second place. Names that do not follow this rule are legitimate, but must be inverted according to Art. 42 b. Species F. nigrescens has a higher cover value than reported for this association.

Because in the original diagnosis the Festuca species belongs to $F$. nigrescens Lam. (Flora Europaea Database), the syntaxon name must be corrected according to Art. 43 .

35. Helianthemo nummularium-Festucetum nigrescentis Matović ex Aćić et al. ass. nov. hoc loco (Orig. Festucetum fallacis Matović 1986 nom. inval.) [Art. 5]

Typus: Matović 1986, Tab. 15, rel. 4 - holotypus hoc loco

36. Centaureo pannonicae-Festucetum orientalis Parabućski ex Aćić et al. ass. nov. hoc loco (Orig. Centaureo pannonicae-Festucetum orientalis Parabućski 1990 nom. inval.) [Art. 5] Typus: Parabućski 1990, Tab. 2, rel. 19 - holotypus hoc loco

37. Agropyro repentis-Festucetum pratensis Veljović 1967

(Orig. Agropyreto-Festucetum pratensis Veljović 1967) [Art. 41b]

Typus: Veljović 1967a, Tab. 1, rel. 12 - lectotypus hoc loco

38. Bromo-Cynosuretum cristati Horvatić 1930 (syn. Cynosuretum cristati Horvatić 1930)

Note: This association was described in Serbia by Gajić $(1954,1961)$, Cincović \& Kojić (1955), Cincović (1959) and Jovanović R. (1965). 
39. Festuco nigrescentis-Cynosuretum cristati Petković et Tatić ex Aćić et al. ass. nov. hoc loco (Orig. Festuco rubrae-Cynosuretum cristati Petković et Tatić 1987 nom. inval.) [Art. 5] Typus: Petković 1981, Tab. 8, rel. 3 - holotypus hoc loco

Note: The name was described in Petković's doctoral thesis (1981) and then published invalidly in the paper by Petković \& Tatić (1987) with a synoptic table only. We have selected a representative relevé from the table in the $\mathrm{PhD}$ thesis and described the habitat of this association.

The association Festuco nigrescentis-Cynosuretum cristati is a mesotrophic pasture developed mainly on south slopes on limestone.

Holotype: Festuca nigrescens 1, Cynosurus cristatus 3, Carum carvi +, Bromus racemosus +, Rhinanthus minor + , Trifolium pratense 1, Lotus corniculatus 1, Stachys officinalis + , Trifolium repens 1 , Colchicum autumnale + , Prunella vulgaris + , Trifolium patens + , Holcus lanatus + , Lolium perenne + , Phleum pratense + , Briza media,+ Anthoxanthum odoratum 1, Euphrasia rostkoviana +, Filipendula hexapetala 1, Agrostis capillaris +, Trifolium montanum 1, Galium verum + , Sanguisorba minor + , Plantago lanceolata + , Linum catharticum + , Ranunculus millefoliatus + , Danthonia alpina + , Achillea setacea + , Hypochoeris radicata + , Brachypodium pinnatum + , Oenanthe fistulosa + , Bromus mollis + , Dactylis glomerata + , Convolvulus arvensis + , Hieracium piloselloides + , Allium carinatum + , Hieracium praealtum ssp. bauhinii + , Centaurea pannonica 1, Ranunculus acris 1, Elymus repens +, Poa badensis +, Medicago sativa ssp. falcata + .

Locality: Southwestern Serbia, Žeđevac (around Tutin), altitude $936 \mathrm{~m}$, aspect S, slope $3^{\circ}$, relevé area $100 \mathrm{~m}^{2}$, limestone.

40. Knautio arvensis-Cynosuretum cristati Gajić 1989 ex Aćić et al. ass. nov. hoc loco

(Orig. Knautio-Cynosuretum cristati Gajić 1989 nom. inval.) [Art. 5]

Typus: Gajić 1989, Tab. 12, rel. 2 - lectotypus hoc loco

Note: The association Knautio-Cynosuretum cristati Blečić et Tatić 1967 was described in Montenegro (Blečić \& Tatić 1967). However, these plant communities are different from the community from Montenegro with the dominant species Knautia purpurea var. montenegrina.

41. Poo trivialis-Cynosuretum cristati JovanovićDunjić ex Aćić et al. ass. nov. hoc loco

(Orig. Poa trivialis-Cynosurus cristatus prov. Jovanović-Dunjić 1955 nom. inval.) [Art. 3b] Typus: Jovanović-Dunjić 1955, table on page 33 , rel. 1 - holotypus hoc loco

42.Potentillo erectae-Cynosuretum cristati Matović 1986 ex Aćić et al. ass. nov. hoc loco

(Orig. Potentilleto-Cynosuretum cristati Matović 1986 nom. inval.) [Art. 5]

Typus: Matović 1986, Tab. 14, rel. 2 - holotypus hoc loco

43. Rhinantho rumelici-Cynosuretum cristati Blečić et Tatić 1960 nomen mutatum propositum (Orig. Alectorolopho-Cynosuretum cristati Blečić et Tatić 1960) [Art. 45]

Typus: Blečić \& Tatić 1960, Tab. 1, rel. 13 - lectotypus hoc loco

\section{Trifolio patentis-Cynosuretum cristati Veljović} 1967

(Orig. Trifolio-Cynosuretum cristati Veljović 1967)

Typus: Veljović 1967a, Tab. 5, rel. 12 - lectotypus hoc loco

45. Plantago lanceolatae-Poetum trivialis Danon et Blaženčić 1965 nomen inversum propositum (Orig. Poeto-Plantaginetum lanceolatae Danon et Blaženčić 1965) [Art. 41a, 42]

Typus: Danon \& Blaženčić 1965, Tab. 2, rel. 1 - lectotypus hoc loco

Note: As mentioned before, if a name of an association is formed from names of two taxa of which one is dominant, that taxon has to appear on the second place. Names that do not follow this rule are legitimate, but must be inverted according to Art. 42. Poa trivialis is the dominant species in this association.

46. Polygono bistortae-Poetum trivialis Pavlović 1951 corr. Aćić et al. ass. hoc loco

(Orig. As. Polygonum bistorta-Poa trivialis Pavlović 1951) [Art. 41b]

Typus: Pavlović 1951, Tab. 10, rel. 2 - lectotypus hoc loco 
47. Gladiolo-Sanguisorbetum officinalis N. Ranđelović et Rexhepi 1984 nomen nudum

Note: The name of the syntaxon is not validly published (Art. 2), as no table or relevé was provided.

48. Armerio rumelicae-Trisetetum flavescentis Rexhepi 1974

(Orig. Armerio-Trisetetum flavescentis Feri 1974) Typus: Rexhepi 1974, Tab. 1, rel. 1 - lectotypus hoc loco

Note: The originally indicated author's name was Feri, although his surname is Rexhepi.

\subsubsection{Cynosurion cristati Tx. 1947}

49. Lolio perennis-Cynosuretum cristati Tx. 1937

Note: The association was described for Serbia in the publication Slavnić (1948) and Parabućski et al. (1986).

\section{Trifolio-Lolietum perennis Krippelová 1967}

Note: The association was described for Serbia in the publication Vučković R. (1985).

\subsection{Trifolio-Hordeetalia Horvatić 1963}

\subsubsection{Trifolion pallidi Ilijanić 1969}

51. Oenantho banaticae-Alopecuretum pratensis Parabućski et Stojanović ex Aćić et al. ass. nov. hoc loco

(Orig. Oenanthe (banatica)-Alopecuretum pratensis Parabućski et Stojanović 1988 nom. inval.) [Art. 5]

Typus: Parabućski \& Stojanović 1988, Tab. 1, rel. 4 - holotypus hoc loco

52. Ranunculo acris-Alopecuretum pratensis $\mathrm{M}$. Vučković ex Aćić et al. ass. nov. hoc loco (Orig. Ranunculo steveni-Alopecuretum pratensis M. Vučković 1991 nom. inval.) [Art. 5] Typus: Vučković M. 1991, Tab. 2, rel. 3 - holotypus hoc loco

Note: The association was described in doctoral thesis of Vučković M. (1988) and later published in a monograph (Vučković M. 1991), but without the nomenclatural type.

53. Trifolio pallidi-Alopecuretum pratensis Cincović 1959

(Orig. Trifolieto-Alopecuretum pratensis Cincović 1959) [Art. 41a]

(syn. Trifolium pallidum-Alopecurus pratensis prov. Cincović 1956)

Typus: Cincović 1959, Tab. 3, rel. 22 - lectotypus hoc loco

Note: The association was first described as provisional (Cincović 1956) and then validly in Cincović (1959).

54. Ornithogalo pyramidale-Trifolietum pallidi M. Vučković ex Aćić et al. ass. nov. hoc loco (Orig. Ornithogalo pyramidale-Trifolietum pallidi M. Vučković nom. inval.) [Art. 5]

Typus: Vučković, M. 1991, Tab. 1, rel. 3 - holotypus hoc loco

Note: The association was described in doctoral thesis of Vučković M. (1988) and later published in a monograph (Vučković M. 1991), but without the nomenclatural type.

\subsubsection{Trifolion resupinati Micevski 1957}

55. Trifolio resupinati-Agrostietum stoloniferae $\mathrm{R}$. Jovanović ex Aćić et al. ass. nov. hoc loco (Orig. Agrostidetum albae prov. R. Jovanović 1965 nom. inval.) [Art. 1, 3b]

Typus: Jovanović R. 1965, Tab. 9, rel. 16 - holotypus hoc loco

Note: This syntaxon was described as provisional in doctoral thesis, so we selected the nomenclatural type and changed the name by adding the differential species Trifolium resupinatum.

The association Trifolio resupinati-Agrostietum stoloniferae is a lowland mesophilous meadow developed on alluvial sediments.

Holotype: Agrostis stolonifera 4, Beckmannia eruciformis + , Carex hirta + , Eleocharis palustris 1, Gratiola officinalis +, Hordeum secalinum +, Inula britannica 1, Funcus articulatus + , Lathyrus latifolius +, Lysimachia nummularia 1, Mentha longifolia 1, Mentha pulegium 2, Poa praten- 
sis + , Poa trivialis 2, Potentilla reptans 2, Prunella vulgaris 1 , Rumex crispus +, Trifolium fragiferum 1, Trifolium hybridum 1, Trifolium repens 2 , Trifolium resupinatum 2, Galium debile 1, Lotus tenuis 1, Carex otrubae 1, Glyceria maxima 1.

Locality: Valley of the river Velika MoravaBukovce, date June 1959, relevé area $100 \mathrm{~m}^{2}$, total cover $100 \%$, alluvial sediments.

56. Stachyo officinalis-Alopecuretum pratensis Veljović 1967 nomen mutatum propositum (Orig. Betonico-Alopecuretum pratensis Veljović 1967) [Art. 45]

Typus: Veljović 1967b, Tab. 2, rel. 3 - lectotypus hoc loco

57. Poo silvicolae-Alopecuretum pratensis R. Jovanović 1972 nomen ineditum [Art. 1]

Note: This association has never been published, but the data was taken from the reports for the mapping project of vegetation of Yugoslavia. The name has not been published in a printed publication and is therefore rendered ineffective (nomen ineditum).

58. Poo trivialis-Alopecuretum pratensis Regel 1925

(homonym Poo trivialis-Alopecuretum pratensis R. Jovanović 1957)

Note: The association was described for Serbia in Jovanović R. (1957).

59. Bromo commutati-Festucetum pratensis B. Jovanović in Jovanović-Dunjić et al. 1986 nomen nudum [Art. 2]

(Orig. Bromo commutati-Festucetum pratensis B. Jovanović 1972 nomen ineditum [Art. 1])

Note: The name of this association was taken from the reports for the mapping of vegetation of Yugoslavia. In the publication of Jovanović R. (1986) it was described with a synoptic table without the nomenclatural type, so the name has not been published with a sufficient original diagnosis. As there is still no singular relevé available, the association remains as nomen nudum.

60. Cynosuro-Caricetum hirtae K. Micevski 1957

Note: The association was described for Ser- bia in Micevski (1968), Jovanović V. (1977, 1979) and Ranđelović (1978).

61. Hordeo-Caricetum distantis K. Micevski 1957

Note: The association was described for Serbia in Micevski (1968), Jovanović V. (1979) and Hundozi (1982).

62. Festuco pratensis-Hordeetum secalini $\mathrm{R}$. Jovanović 1957

(Orig. Festuceto-Hordeetum secalini R. Jovanović 1957) [Art. 41b]

Typus: Jovanović, R., 1957, Tab. 2, rel. 18 - lectotypus hoc loco

63. Festuco pratensis-Brometum racemosi Danon et Blaženčić in Mišić et al. 1978

(Orig. Festuco-Brometum racemosi Danon et Blaženčić 1975)

Typus: Mišić et al. 1978, Tab. 43, rel. 2 - lectotypus hoc loco

64. Lathyro pratensis-Galietum veri Veljović 1967 (Orig. Lathyro-Galietum veri Veljović 1967)

Typus: Veljović, 1967b, Tab. 3, rel. 4 - lectotypus hoc loco

\section{Poo-Trifolietum fragiferi K. Micevski 1968}

Note: The association was described for Serbia in Ranđelović (1978) and Hundozi (1980).

66. Trifolietum nigrescentis-subterranei $\mathrm{K}$. Micevski 1957

Note: The association was described for Serbia in Micevski (1978), Ranđelović (1978) and Jovanović V. (1979).

67. Trifolietum resupinati Veljović ex Aćić et al. ass. nov. hoc loco

(Orig. Trifolietum resupinati prov. Veljović 1967 nom. inval.) [Art. 3b]

Typus: Veljović 1967b, Tab. 1, rel. 4 - holotypus hoc loco

68. Trifolietum resupinati-balansae K. Micevski 1959

Note: The association was described for Serbia in Micevski (1968), Ranđelović (1978), Jovanović V. (1979) and Hundozi (1980). 


\subsubsection{Trifolio-Ranunculion pedati Slavnić 1948}

69. Cynosuro-Alopecuretum pratensis Parabućski 1985 nomen ineditum [Art. 1]

Note: This association has never been published, but the name was taken from the reports for the mapping of vegetation of Yugoslavia and was later published in Prodromus of vegetation of Vojvodina (Parabućski et al. 1986).

70. Rorippo kerneri-Alopecuretum pratensis Purger ex Aćić et al. ass. nov. hoc loco

(Orig. Roripo kerneri- Alopecuretum pratensis Purger 1993 nom. inval.) [Art. 1, 5]

Typus: Purger 1993, Tab. 2, rel. 1 - holotypus hoc loco

Note: This association was published in a master thesis, so we selected a representative relevé together with the description of its habitat.

The association Rorippo kerneri-Alopecuretum pratensis is a secondary grassland developed on slightly salinized soil on loess.

Holotype: Alopecurus pratensis 4, Carex melanostachya +, Carex pallescens + , Carex vulpina +, Gratiola officinalis 2, Lysimachia nummularia 1, Oenanthe silaifolia + , Polygonum lapathifolium + , Poa pratensis 1, Potentilla reptans + , Ranunculus polyanthemos 1 , Rorippa austriaca + , Rorippa kerneri 1 , Taraxacum officinale + .

Locality: Vojvodina, western Bačka, Doroslov, altitude $88 \mathrm{~m}$, relevé area $25 \mathrm{~m}^{2}$, total cover $80 \%$, slightly salinized soil on loess.

71. Trifolio angulati-Alopecuretum pratensis Parabućski ex Aćić et al. ass. nov. hoc loco (Orig. Trifolio angulati-Alopecuretum pratensis Parabućski 1985 nom. inval.) [Art. 1, 5] Typus: Purger 1993, Tab. 3, rel. 2 - holotypus hoc loco

Note: The name of this association was taken from the reports for vegetation mapping of $\mathrm{Yu}$ goslavia and Prodromus of vegetation of Vojvodina. In her master thesis Purger (1993) described this association without the nomenclatural type, so we selected a representative relevé and described the habitat of this association.

The association Trifolio angulati-Alopecuretum pratensis is a salinized pannonian meadow developed on loess.
Holotype: Alopecurus pratensis 1, Carex divisa + , Gratiola officinalis + , Lotus corniculatus + , Lysimachia nummularia + , Oenanthe silaifolia 3 , Potentilla reptans + , Ranunculus polyanthemos + , Rorippa kerneri + , Rumex crispus + , Trifolium angulatum 1, Trifolium dubium + , Trifolium striatum + , Vicia tetrasperma + , Scorzonera cana + , Myosotis ramosissima + .

Locality: Vojvodina, western Bačka, Doroslov, altitude $88 \mathrm{~m}$, relevé area $25 \mathrm{~m}^{2}$, total cover $90 \%$, slightly saline loess.

72. Poo pratensis-Alopecuretum pratensis $\mathrm{R}$. Vučković ex Aćić et al. ass. nov. hoc loco (Orig. Poeto-Alopecuretum pratensis halophyticum Vučković 1982 nom. inval.) [Art. 5, 34a] Typus: Vučković R. 1982, Tab. 1, rel. 3 - holotypus hoc loco

\section{Peucedano officinalis-Asteretum sedifolii Soó} 1947

(syn. Peucedano-Asteretum punctati Soó 1947, Peucedano-Galatelletum punctati Máthé, Tallós et Zólyomi 1967)

Note: The association was described for Serbia in Parabućski \& Stojanović (1978), Parabućski (1979) and Vučković R. (1985).

\section{Ranunculetum pedati Slavnić 1948} (Orig. Ranunculetum pedati Slavnić 1942)

Note: Only a synoptic table was published (Slavnić 1948). No single relevé is available as the nomenclatural type. Although the author states the year 1942, the year of printing was 1948.

75. Trifolietum subterranei Slavnić 1948 (Orig. Trifolietum subterranei Slavnić 1942) Typus: Parabućski 1979, Tab. 2, rel. 11- neotypus hoc loco

Note: Acording to Article 21 (ICPN), when the original diagnosis of an association contains only a synoptic table but no single relevé, then a neotype must be established. The first effectively published establishment of a neotype must be followed and the neotype should be taken from the same geographical area as the relevés of the synoptic table. We selected a relevé from the same geographical area as the neotype from Parabućski (1979). 
Although the author states the year 1942 the year of printing was 1948 .

The association Trifolietum subterranei is a steppic grassland developed on saline solonetz soil type.

Neotype: Achillea collina 1, Alopecurus pratensis +, Bromus hordeaceus 2, Cerastium semidecandrum 1, Festuca pseudovina 1, Poa angustifolia 1, Ranunculus pedatus 1, Trifolium subterraneum 4, Vicia tetrasperma 1, Taraxacum officinale +, Poa bulbosa f. vivipara 1, Cardaria draba 1.

Locality: Vojvodina, Bačka, Đurđevo, altitude $80 \mathrm{~m}$, total cover $80 \%$, solonetz.

1.4 Potentillo-Polygonetalia avicularis Tx. 1947 (syn. Agrostetalia stoloniferae Oberd. 1967)

\subsubsection{Potentillion anserinae Tx. 1947}

(syn. Agropyro-Rumicion crispi Nordh. 1940, Agrostion albae (stoloniferae) Soo (1933) 1971)

\section{Oenantho silaifoliae-Agrostietum stoloniferae}

R. Vučković ex Aćić et al. ass. nov. hoc loco (Orig. Agrostetum albae (Ujv. 1941) pannonicum R. Vučković 1985 nom. inval.) [Art. 1, 5, 34a] Typus: Vučković R. 1985, Tab. 21, rel. 3 - holotypus hoc loco

The association Oenantho silaifoliae-Agrostietum stoloniferae is a Pannonian mesic grassland developed on salinized soils.

Holotype: Agrostis stolonifera 4, Alopecurus pratensis 1 , Beckmannia eruciformis 1, Eleocharis palustris +, Elymus repens +, Myosotis laxa ssp. caespitosa + , Oenanthe silaifolia 1, Ranunculus lateriflorus +, Rorippa kerneri + , Rumex crispus +

Locality: Vojvodina, valley of the river Tamiš, between Sečanj and Jarkovac, relevé area $25 \mathrm{~m}^{2}$, total cover $100 \%$.

Note: This association was published in doctoral thesis, so we selected a representative relevé and described the habitat of this association. The name is illegitimate and must be rejected because it contains an epithet in the nominative case that indicates a geographical property. We selected the species Oenanthe silaifolia because it is a character species of the association.
77. Hordeo histricis-Agrostietum stoloniferae R. Vučković ex Aćić et al. ass. nov. hoc loco (Orig. Halo-Agrostietum albae R. Vučković 1985 nom. inval.) [Art. 1, 5, 34a]

Typus: Vučković R. 1985, Tab. 22, rel. 5 - holotypus hoc loco

The association Hordeo histricis-Agrostietum stoloniferae is a Pannonian halophytic grassland developed on saline solonetz soil type.

Holotype: Agrostis stolonifera 4, Alopecurus pratensis + , Eleocharis palustris 1, Inula britannica + , Limonium gmelinii +, Lolium perenne + , Polygonum aviculare + , Rorippa kerneri + , Trifolium angulatum + , Scorzonera cana + , Hordeum hystrix 1 .

Locality: Vojvodina, valley of the river Tamiš, between Boka and Tomaševac, relevé area $25 \mathrm{~m}^{2}$, total cover $100 \%$.

Note: This association was published in doctoral thesis, so we selected a representative relevé and described the habitat of this association. The name is illegitimate and must be rejected because it contains an epithet in the nominative case that indicates an ecological property. We selected the species Hordeum hystrix because it occurs with constancy III.

\section{Trifolio fragiferi-Agrostietum stoloniferae Mar-} ković 1978

Note: The association was described for Serbia in Butorac $(1989,1992)$ and Stojanović et al. (1997).

\section{Rumici crispi-Agrostietum stoloniferae Moor} 1958

(syn. Rorippo silvestris-Agrostietum stoloniferae (Moor 1958) Oberdorfer et T. Müller in T. Müller 1961)

Note: The association is mentioned for Serbia in Parabućski et al. (1986) and Stojanović et al. (1997), but without a table or relevé.

\section{Ranunculo repentis-Alopecuretum geniculati} Tüxen 1937

(syn. Rumici crispi-Alopecuretum geniculati $\mathrm{R}$. Tx. 1950)

Note: The association was described for Serbia in Rauš et al. (1980) and Parabućski et al. (1986). 
81. Limonio gmelini-Alopecuretum pratensis $\mathrm{R}$. Vučković ex Aćić et al. ass. nov. hoc loco (Orig. Alopecuretum pratensis (Reg. 25, Nowin. 28) panonnicum R. Vučković 1985 nom. inval.) [Art. 1, 5, 34a])

Typus: Vučković R. 1985, Tab. 23, rel. 3 - holotypus hoc loco

The association Limonio gmelini-Alopecuretum pratensis is a Pannonian halophytic grassland developed on salinized soils.

Holotype: Allium vineale + , Alopecurus pratensis 5, Cynodon dactylon,+ Festuca pseudovina 1, Limonium gmelinii 1, Poa pratensis + , Rorippa kerneri + , Rumex crispus + , Trifolium angulatum 1, Taraxacum officinale + , Scorzonera cana + .

Locality: Vojvodina, valley of the river Tamiš, relevé area $25 \mathrm{~m}^{2}$, total cover $100 \%$.

Note: This association was published in doctoral thesis, so we selected a representative relevé and describe the habitat of this association. The name is illegitimate and must be rejected because it contains an epithet in the nominative case that indicates a geographical property. We select the species Limonium gmelinii because it occurs with constancy IV.

82. Funco inflexi-Menthetum longifoliae Lohmeyer ex Oberdorfer 1957

Note: The association was described for Serbia in Parabućski et al. (1986) and Butorac (1989).

83. Agropyro repentis-Poetum angustifoliae Babić 1972

(Orig. Agropyro-Poetum angustifoliae Babić 1971)

Typus: Babić 1972, Tab. 2, rel. 5 - lectotypus hoc loco

Note: The association was described in doctoral thesis of Babić (1965) and then validly published in his paper (Babić 1972). Although the author states the year 1971, the year of printing was 1972.

\section{Potentilletum anserinae Rapaics 1927}

Note: The association was mentioned for Serbia in Parabućski et al. (1986) and Stojanović et al. (1997), but without a table or relevé.

\subsection{Poo alpinae-Trisetetalia Ellmauer et Muci- na 1993}

\subsubsection{Pancicion serbicae Lakušić 1966}

85. Pancicio-Centauretum nervosae prov. Lakušić 1970 nomen nudum

Note: The name of the syntaxon is not validly published (Art. 2) as there is no published phytocoenological table or relevé (Lakušić 1970). The association was mentioned for Serbia in Amidžić (2003), but without a phytocoenological table.

\section{Festuco nigrescentis-Pancicietum serbicae Stan-} ković-Tomić 1970

(Orig. Festuco-Pančićietum Stanković-Tomić 1970) [Art. 41a]

Typus: Stanković-Tomić 1970, Tab. 1, rel. 2 lectotypus hoc loco

87. Ranunculo-Pancicietum serbicae R. Lakušić 1966

(Orig. Ranunculo-Pančićietum serbicae Lakušić 1966)

Note: The association was described for Montenegro (Lakušić 1966) and mentioned for Serbia in Amidžić (2003), but without a phytocoenological table.

\section{CONCLUSIONS}

The present work, based on an overview of comprehensive phytosociological literature, considers all associations, alliances and orders that have been included in the class Molinio-Arrhenatheretea in Serbia. The list of corrected and typified syntaxa will allow for in-depth vegetation analyses of particular syntaxa, further mapping of grassland vegetation, integration of communities into the system of the EU Habitat Directive, and may serve as the basis for the establishment of The Red list of Habitats in Serbia. This revision could also enable a comparison and validation of plant communities of Serbia in a broader transnational space.

We believe that the present study is a stepping stone towards a consistent classification of plant communities in Serbia and their appropriate positioning within a syntaxonomical scheme of Europe. 


\section{ACKNOWLEDGEMENTS}

We thank prof. Ladislav Mucina for allowing us to use the unpublished data from the EuroVegChecklist. This work was supported by the Ministry for Education and Science of Serbia, Grant TR 31057, ON 173030 and the Bilateral Grant Slovenia-Serbia No. 12-13-035.

\section{REFERENGES}

Amidžić, L. 2003: Visokoplaninska vegetacija. In: Metohijske Prokletije - prirodna i kulturna baština. Zavod za zaštitu prirode Srbije, Beograd, 474 pp.

Aćić S., Petrović M., Dajić Stevanović, Z. \& Šilc U. (2012): Vegetation database Grassland vegetation in Serbia. In: Dengler, J., Chytrý, M., Ewald, J., Finckh, M., Jansen, F., Lopez-Gonzalez, G., Oldeland, J., Peet, R. K. \& Schaminée, J. H. J. (2012) (eds.): Vegetation databases for the $21^{\text {st }}$ century. Biodiversity \& Ecology 4: 418.

Aćić, S., Šilc, U., Vrbničanin, S., Cupać, S., Topisirović, G., Stavretović, N. \& Dajić Stevanović, Z. 2013: Grassland communities of Stol mountain (eastern Serbia): Vegetation and environmental relationships. Archives of Biological Sciences 65 (1): 211-227.

Adamović, L. 1909: Die Vegetationsverhältnisse der Balkanländer (Mösische Länder) umfassend Serbien, Altserbien, Bulgarien, Ostrumelien, Nordthrakien und Nordmazedonien. In: Engler, A. \& Drude, O. (ed.): Vegetation der Erde 11. Wilhelm Engelmann, Leipzig, 12-23.

Babić, N. 1955: Nizinske livade u Podunavlju. Rad Vojvođanskih muzeja 4: 155-156.

Babić, N. 1965: Močvarna i livadska vegetacija Koviljskog rita (fitocenološka studija). Doctoral thesis. PMF Univerziteta u Beogradu, Beograd, 343 pp.

Babić, N. 1972: Močvarna i livadska vegetacija Koviljskog rita. Matica srpska, Zbornik za prirodne nauke 41: 19-87.

Barkmann, J., Moravec, J. \& Rauschert, S. 1976: Code of phytosociological nomenclature, 1 st edition. Vegetatio 32: 131-185.

Barkmann, J., Moravec, J. \& Rauschert, S 1986: Code of phytosociological nomenclature, 2 nd edition. Vegetatio 67: 145-195.

Blečić, V. \& Tatić, B. 1960: Beitrag zur Kenntniss der Vegetation Ostserbiens. Glasnik Bota- ničkog zavoda i bašte Univerziteta u Beogradu I (V) 2: 119-130.

Blečić, V. \& Tatić, B. 1967: Association du Cynosure à crètes dans les prairies de hautes vallées de Montenegro. Glasnik Botaničkog zavoda i bašte Univerziteta u Beogradu 2(1-4): 131-140.

Braun-Blanquet, J. 1928: Pflanzensoziologie. Grundzüge der Vegetationskunde. Springer, Berlin, 330 pp.

Butorac, B. 1989: Vegetacija Sremskog lesnog platoa. Doctoral thesis. Prirodno-matematički fakultet, Univerzitet u Novom Sadu, 348 pp.

Butorac, B. 1992: Vegetacija fruškogorskog lesnog platoa, Monografija Fruške Gore. Matica srpska, Odeljenje za prirodne nauke, Novi Sad, 163 pp.

Butorac, B. \& Hulo, I. 1993: Contribution to Knowledge of Marsch Meadows around the Kereš River. Book of Abstracts of XXIV Tiszakutató Ankét, Szegedi Ökologógiai Napok: 6, Segedin.

Cincović, T. \& Kojić, M. 1955: Livadske fitocenoze Maljena. Zbornik radova Poljoprivrednog fakulteta 3 (1): 113-118.

Cincović, T. \& Kojić, M. 1956: Neki tipovi livada i pašnjaka na Divčibarama. Zbornik radova Poljoprivrednog fakulteta, 4 (2): 37-58.

Cincović, T. 1956: Tipovi livada u Posavini. Zbornik radova Poljoprivrednog fakulteta, IV (1): $1-25$.

Cincović, T. 1959: Livadska vegetacija u rečnim dolinama zapadne Srbije. Doctoral thesis, Univerzitet u Beogradu, Poljoprivredni fakultet, Beograd, 62 pp.

Corley, V., Crundwell, G., Dull, R., Hill, O. \& Smith, E. 1981: Mosses of Europe and the Azores. Journal of Bryology 11: 609-689.

Čapaković, J. 1979: Zajednica Carex gracilis-Poa palustris u Petrovaradinskom ritu. Matica srpska, Zbornik za prirodne nauke 57: 207-220.

Danon, J. 1960: Fitocenološka ispitivanja livada tipa Agrostidetum vulgaris i Poterieto-Festucetum vallesiaceae u okolini Krivog vira. Archives of biological sciences 12 (1-2): 1-9.

Danon, J., Blaženčić, Z. 1965: Ekološka analiza livadskih zajednica vlažnih i poluvlažnih staništa Stare planine. Archives of biological sciences 17(1-2): 101-112.

Dinić, A., Marković, A. \& Šijak, M. 2010: Igor Andrejević Rudski-On the occasion of the 70-year anniversary of the first phytocoenological research in Serbia. Bulletin of the Natural History Museum 3: 189-221. 
Flora Europea Database: Royal Botanic Garden Edinburgh. http://rbg-web2.rbge.org.uk/FE/ fe.html

Gajić, M. 1954: Šumske i livadske fitocenoze Kosmaja. Arhiv bioloških nauka 6 (1-2): 1-16.

Gajić, M. 1961: Fitocenoze i staništa planine Rudnik i njihove degradacione faze. Glasnik Šumarskog fakulteta 23: 3-114.

Gajić, M. 1986: Flora i vegetacija SubotičkoHorgoške peščare. Šumarski fakultet Beograd, Šumsko gazdinstvo Subotica, Subotica, 495 pp.

Gajić, M. 1989: Flora i vegetacija Golije i Javora. Šumarski fakultet, Beograd i Šumarstvo Golija, Ivanjica, 592 pp.

Gajić, M., Kojić, M., Karadžić, D., Vasiljević, M. \& Stanić, M. (1992): Vegetacija Nacionalnog parka Tara. Šumarski fakultet, Beograd, Nacionalni park Tara, Bajina Bašta, 288 pp.

Hundozi, B. 1980: Vegetacija nizinskih livada na Kosovu. Doctoral thesis, Prirodoslovno-matematički fakultet, Zagreb, 173 pp.

Hundozi, B. 1982: Hordeo-Caricetum distantis Mic. 1957 u vegetaciji Kosova. Buletini shkencave i FSHMN-Prishtinë (Zbornik radova PMFPriština) 8: 189-202.

Jovanović, B., Tomić, Z., Purić, O. \& Atanacković, B. 1969: Vegetacijska karta sa ekološko-fitocenološkom analizom (komentarom) područja "Niš 2 - Pirot 1 - Pirot 2". Institut za biološka istraživanja "Siniša Stanković, Beograd, 3 pp.

Jovanović, B. \& Jovanović, R. 1976: Prodromus biljnih zajednica SR Srbije (bez pokrajina). Institut za biološka istraživanja "Siniša Stanković", Beograd, 74 pp.

Jovanović, B., Jovanović, R. \& Zupančić, M. (eds) 1986: Karta prirodne potencijalne vegetacije Jugoslavije, Komentar karte M $1: 1.000$ 000 . Naučno veće vegetacijske karte Jugoslavije, Ljubljana, 122 pp.

Jovanović-Dunjić, R. 1955: Tipovi pašnjaka i livada Suve Planine. Srpska akademija nauka, Institut za ekologiju i biogeografiju, Zbornik radova 6(2): 1-104.

Jovanović, R. 1957: Tipovi dolinskih livada Jasenice. Archives of biological sciences 9(1-4): $1-14$.

Jovanović-Dunjić, R. 1965: Tipologija, ekologija i dinamika močvarne i livadske vegetacije u dolini Velike Morave. Doctoral thesis, Univerzitet u Beogradu, PMF, Beograd, 399 pp.

Jovanović-Dunjić, R. 1979: Uporedna analiza promena u sastavu i strukturi zajednice Sparganio-Eriophoretum latifolii R. Jov. 1976 u perio- du 1959-1973-1977. In: Rauš, Đ. (ed.): Drugi Kongres ekologa Jugoslavije, Savez Društava ekologa Jugoslavije, Zagreb, 1: 585-595.

Jovanović-Dunjić, R. 1983: Prilog proučavanju sastava i strukture livadske zajednice Arrhenatheretum elatioris Br.-Bl. na području Velikog Jastrepca. Archives of biological sciences 35(1-2): 51-65.

Jovanović-Dunjić, R., Stefanović, K., Popović, R. \& Dimitrijević, J. 1986: Prilog poznavanju livadskih ekosistema na području Velikog Jastrebca. Glasnik Instituta za botaniku i Botaničke bašte Univerziteta u Beogradu 20: 7-31.

Jovanović-Dunjić, R. \& Jovanović, S. 1991: Pregled zajednica livada, pašnjaka i planinskih tresava na području Nacionalnog parka Tara. Glasnik Instituta za botaniku i Botaničke bašte Univerziteta u Beogradu 23: 69-75.

Jovanović, V. 1977: Planina Kukavica u jugoistočnoj Srbiji i vegetacija njenog severnog dela. Leskovački Zbornik 17: 271-299.

Jovanović, V. 1979: Livadska vegetacija jugoistočne Srbije (planina Radan, Goljak, deo Kukavice i njihova okolina). Doctoral thesis. PMF Univerzitet u Novom Sadu, 139 pp.

Kojić, M. \& Ivanović, M. 1953: Fitocenološka istraživanja livada na južnim padinama Maljena. Zbornik radova Poljoprivrednog fakulteta 1: 1-22.

Kojić, M., Popović, R. \& Karadžić, B. 1998: Sintaksonomski pregled vegetacije Srbije. Institut za biološka istraživanja "Siniša Stanković", Beograd, 220 pp.

Kojić, M., Mrfat-Vukelić, S., Dajić, Z. \& ĐorđevićMilošević, S. 2004. Livade i pašnjaci Srbije I. Pregled i vrednovanje dosadašnjih fitocenoloških istraživanja i pravci daljih istraživanja. Institut za istraživanja u poljoprivredi „Srbija“, Beograd, 92 pp.

Lakušić, R. 1966: Vegetacija livada i pašnjaka na planini Bjelasici. Godišnjak Biološkog Instituta Univerziteta u Sarajevu 19: 25-186.

Lakušić, R. 1970: Florističke rijetkosti i vegetacijske zakonitosti planine Hajle (2400 m. s. m.) (Die Vegetationsgliederung des Hajla Gebirges -2400 m. s. m.). Glasnik Republičkog zavoda za zaštitu prirode i Prirodnjačkog muzeja u Titogradu 3: 49-66.

Lakušić, D. \& Sabovljević, M. 2005: Fitocenološka klasifikacija staništa. In: Lakušić, D. (ed.): Staništa Srbije, Rezultati projekta "Harmonizacija nacionalne nomenklature u klasifikaciji staništa sa standardima međunarodne zajed- 
nice”, Institut za Botaniku i Botanička Bašta "Jevremovac", Biološki fakultet, Univerzitet u Beogradu, Ministarstvo za nauku i zaštitu životne sredine Republike Srbije, http://habitat. bio.bg.ac.rs/nacionalne_klasifikacije_stanista.htm

Lakušić, D., Blaženčić, J., Ranđelović, V., Butorac, B., Vukojičić, S., Zlatković, B., Jovanović, S., Šinžar-Sekulić, J., Žukovec, D., Ćalić, I. \& Pavićević, D. 2005: Staništa Srbije - Priručnik sa opisima i osnovnim podacima. In: Lakušić, D. (ed.): Staništa Srbije, Rezultati projekta "Harmonizacija nacionalne nomenklature u klasifikaciji staništa sa standardima međunarodne zajednice", Institut za Botaniku i Botanička Bašta "Jevremovac", Biološki fakultet, Univerzitet u Beogradu, Ministarstvo za nauku i zaštitu životne sredine Republike Srbije, 684 pp., http://habitat.bio.bg.ac.rs/

Matović, M. 1986: Monografija o biljnom pokrivaču okoline Prijepolja. Glas Polimlja, Prijepolje, 166 pp.

Micevski, K. 1968: Livadska vegetacija na Kosovo pole. Godišen zbornik na Prirodno-matematički fakultet na Univerzitetot vo Skopje, Biologija 20: 135-146.

Mišić, V., Jovanović-Dunjić, R., Popović, M., Borisavljević, Lj., Antić, M., Dinić, A., Danon, J. \& Blaženčić, Ž. 1978: Biljne zajednice i staništa Stare planine. Srpska akademija nauka i umetnosti, Posebna izdanja 511, Odeljenje prirodno-matematičkih nauka 49: 1-389.

Mucina, L., Bültman, H., Dierssen, K., Theurillat, J.-P., Dengler, J., Čarni, A., Šumberová, K., Raus, T., Di Pietro, R., Gavilán Garcia, R., Chytrý, M., Iakushenko, D., Schaminée, J. H. J., Bergmeier, E., Santos Guerra, A., Daniëls, F. J. A., Ermakov, N., Valachovič, M., Pigantti, S., Rodwell, J. S., Pallas, J., Capelo, J., Weber, H. E., Lysenko, T., Solomeshch, A., Dimopoulos, P., Aguiar, C., Freitag, H., Hennekens, S. M. \& Tichý, L. 2013: Vegetation of Europe: Hierarchical floristic classification system of plant, lichen, and algal communities. Applied Vegetation Science (in review).

Mucina, L., Grabherr, G. \& Ellmauer, T. (eds.) 1993: Die Pflanzengesellschaften Österreichs. Teil I: Anthropogene Vegetation. Gustav Fischer Verlag, Jena, 578 pp.

Parabućski, S. \& Stojanović, S. 1978: Neke ekološke i cenološke karakteristike vrste Scilla autumnalis L. u Bačkoj. Matica srpska, Zbornik za prirodne nauke 55: 149-154.
Parabućski, S. 1979: Zajednice Peucedano-Asteretum punctati Soó i Trifolietum subterranei Slavnić na nekim lokalitetima Bačke i njihov sintaksonomski položaj. Matica srpska, Zbornik za prirodne nauke 56: 17-43.

Parabućski, S., Pekanović, V. \& Crnčević, S. 1982: Lotus siliquosus L. (Fabaceae) na nekim lokalitetima Bačke. Biosistematika 8(1): 43-47.

Parabućski, S., Stojanović, S., Butorac, B. \& Pekanović, V. 1986: Prodromus vegetacije Vojvodine. Zbornik za prirodne nauke Matice srpske 71: 5-40.

Parabućski, S. \& Stojanović, S. 1988: Oenanthe (banatica)-Alopecuretum pratensis ass. nova. Zbornik Matice srpske za prirodne nauke 74: 71-78.

Parabućski, S. \& Butorac, B. 1988: Zajednica Molinietum coeruleae $\mathrm{W}$. Koch $1926 \mathrm{u}$ vegetaciji Bačke. Zbornik Matice srpske za prirodne nauke 74: 111-121.

Parabućski, S. 1990: Neke odlike dolinskih livada na području Vojvodine. Zbornik Matice srpske za prirodne nauke 79: 107-118.

Pavlović, Z. 1951: Vegetacija planine Zlatibor. Zbornik radova Instituta za ekologiju i biogeogeografiju SAN, 2: 115-182.

Petković, B. 1981: Livadska vegetacija Tutinskog regiona. Doctoral thesis, Prirodno-matematički fakultet Univerziteta u Beogradu, 209 pp.

Petković, B. 1983: Močvarna vegetacija na području Tutina. Glasnik Instituta za botaniku i Botaničke bašte Univerziteta u Beogradu 17: 61-102.

Petković, B. 1985: Brdske livade i pašnjaci na području Tutina. Glasnik Instituta za botaniku i Botaničke bašte Univerziteta u Beogradu 19: 175-189.

Petković, B. \& Tatić, B. 1987: Nova zajednica vijuka i krestca (Festuco rubrae-Cynosuretum cristati) sa područja Tutina. Glasnik Instituta za botaniku i Botaničke bašte Univerziteta u Beogradu 21: 49-56.

Petković, B., Krivošej, Z. \& Veljić, M. 1996: Selaginello-Eriophoretum latifoli-ass. nova sa planine Ošljak (Srbija, Kosovo). Glasnik Instituta za Botaniku i Botaničke bašte Univerziteta u Beogradu 30: 89-95.

Purger, D. 1993: Vegetacija u okolini Doroslova (zapadna Bačka). Master thesis, PMF Univerzitet u Novom Sadu, 51 pp.

Ranđelović, N. 1978: Fitocenološko-ekološke karakteristike brdskih travnjaka jugoistočne Srbije. Doctoral thesis, Prirodoslovno-mate- 
matički fakultet, Univerzitet u Zagrebu, Zagreb, $143 \mathrm{pp}$.

Ranđelović, N. \& Rexhepi, R. F. 1980: Prodromusi i fitocenozave te Kosoves/Prodromus biljnih zajednica Kosova. Biotehnika 8(3-4): 213-222.

Ranđelović, N. \& Rexhepi, F. 1984: Livadska i pašnjačka vegetacija Koritnika. Bilten Društva ekologa Bosne i Hercegovine B2(1): 271-274. (III Kongres ekologa Jugoslavije, Sarajevo 1984, Radovi i rezimea 1).

Ranđelović, V. \& Zlatković, B. 1994: Vegetacija sveze Calthion u jugoistočnoj Srbiji. Ekologija 28(1-2)-29(1-2): 19-31.

Ranđelović, V. 2002: Flora i vegetacija Vlasinske visoravni. Doctoral thesis, Univerzitet u Beogradu, Biološki fakultet, Beograd, 477 pp.

Ranđelović, V. \& Zlatković, B. 2010: Flora i vegetacija Vlasinske visoravni. Prirodno-matematički fakultet, Univerzitet u Nišu, Niš, 448 pp.

Rauš, Đ., Šegulja, N. \& Topić, J. 1980: Vegetacija bara i močvara u šumama jugozapadnog Srijema. Matica srpska, Zbornik za prirodne nauke 58: 17-51.

Rexhepi, F. 1974: Vegjetacioni i livadheve bregore të Novo Bërdos/Vegetacija brdskih livada Novog Brda. Biotehnika 2(4): 454-461.

Rexhepi, F. \& Ranđelović, N. 1984: Šumska vegetacija Koritnika. Bilten Društva ekologa Bosne i Hercegovine B2(1): 265-270. (III Kongres ekologa Jugoslavije, Sarajevo, radovi i rezimea 1.)

Rudskij, I. A. 1934: O vegetacii gory Ošljaka. [On vegetation of Mt. Ošljak]. Zapiski Russkago Naučnago Instituta v Belgrade 10: 193-202.

Rudski, I. 1938: Biljne zajednice na visokim planinama Južne Srbije. [Plant associations in high mountains of southern Serbia]. Šumarski list 62(12): 611-623.

Sarić, M. R. (ed.) 1984: Vegetacija SR Srbije 1. Srpska akademija nauka i umetnosti, Beograd, $408 \mathrm{pp}$.

Slavnić, Ž. 1948: Slatinska vegetacija Vojvodine. Arhiv za poljoprivredne nauke i tehniku 3(4): 76-142.

Stanković-Tomić, K. 1970: Prilog poznavanju livadske vegetacije Mokre planine II (Kosovo). Zbornik filozofskog fakulteta u Prištini 7: 151-156.

Stjepanović-Veseličić, L. 1953: Vegetacija Deliblatske peščare SANU, Posebna izdanja knjiga CCXVI, Institut za ekologiju i biogeografiju 4, Beograd, 113 pp.
Stojanović, S. 1983: Vegetacija Titelskog brega. Matica srpska, Zbornik za prirodne nauke 65: $5-51$.

Stojanović, S. 1986: Elementi dolinskih livada u vegetaciji Titelskog brega. In: Mihaljev, I. \& Vučić, N. (eds): Čovek i biljka, Zbornik radova sa naučnog skupa, Matica srpska, Odeljenje za prirodne nauke, Novi Sad, pp. 403-410.

Stojanović, S., Vučković, M., Radulović, S. \& Lazić, D. 1997: Recentna livadska vegetacija pašnjačkog karaktera Agrostietalia stoloniferae Oberd. 1967 na području Vojvodine. Zbornik radova Prirodno-matematičkog fakulteta, Novi Sad, Serija za biologiju 26: 44-49.

Tatić, B. 1969: Flora i vegetacija Studene planine kod Kraljeva. Glasnik Botaničkog zavoda i Botaničke bašte IV (1-4): 27-72.

Tatić, B., Veljović, V., Petković, B. Stefanović M. \& Radotić, S. 1988: Ass. Lathyreto-Molinietum coeruleae-nova zajednica livadske vegetacije sa Pešterske visoravni-jugozapadna Srbija. Glasnik Instituta za Botaniku i Botaničke Baste Univerziteta u Beogradu 12: 31-38.

Veljović, V. 1967a: Vegetacija okoline Kragujevca. Glasnik Prirodnjačkog muzeja u Beogradu B 22: 1-109.

Veljović, V. 1967b: Dolinske livade Gruže. Glasnik Prirodnjačkog muzeja u Beogradu B 22: 111-126.

Vučković, R. 1982: Jedna nova asocijacija sveze Festucion pseudovinae Soo 1933. Ekologija 17 (1): 15-23.

Vučković, R. 1985: Fitocenoze slatinske vegetacije istočnog Potamišja, njihova produkcija i hranljiva vrednost. Doctoral thesis, Prirodnomatematički fakultet Univerziteta u Beogradu, $220 \mathrm{pp}$.

Vučković, M. 1988: Livadska i livadsko-stepska vegetacija Vršačkih planina. Doctoral thesis, Prirodno-matematički fakultet Novi Sad, 117 pp.

Vučković, M. 1991: Livadska i livadsko-stepska vegetacija Vršačkih planina. Matica srpska, Novi Sad, 95 pp.

Zupančič, M. (ed.) 1986: Prodromus phytocoenosum Jugoslaviae ad mappam vegetationis $1: 200.000$. Naučno veće vegetacijske karte Jugoslavije, Bribir-Ilok, 46 pp.

Received 1. 8. 2013 Revision received 10. 11. 2013 Accepted 12. 11. 2013 